\title{
Visibilidade de objetos educacionais desenvolvidos pelo Laboratório de Tecnologia Educacional (LTE) em cinco plataformas de distribuição de conteúdo digital
}

\begin{abstract}
Eduardo Galembeck
Juan Carlos Vega Garzon

Resumo

O presente estudo é uma análise da visibilidade dada a 196 conteúdos digitais desenvolvidos pelo LTE em cinco plataformas de distribuição de conteúdo digital. $O$ estudo revela que a visibilidade dada ao material publicado no Portal do Professor difere estatisticamente de forma significativa em relação às outras plataformas estudadas, que não diferem entre si. Os softwares desenvolvidos pelo LTE são mais acessados que áudios, vídeos e documentos. Essa visibilidade alcançada com softwares, aliada à projeção de crescimento de usuários de dispositivos móveis como Tablets e Smartphones apontam para um cenário bastante promissor para o desenvolvimento desta tecnologia com finalidades educacionais e de divulgação científica.
\end{abstract}

Palavras-chave: Biblioteca Digital de Ciências, Google Play, Portal do Professor, Apple App Store, YouTube.

\section{Abstract}

This study is a visibility analysis of 196 digital content developed by LTE in five digital content distribution platforms. The study reveals that there are statistically significant difference between materials published on Portal do Professor and the other platforms. The other four platforms studied don't show significant difference among them. Software's developed by LTE were more accessible to audios, videos and documents; this visibility, coupled with the projected growth of users of mobile devices such as Tablets and Smartphones are a very promising scene for development of this technology for educational and science communication.

Keywords: Digital Library of Science, Google Play, Teacher Portal, Apple App Store, YouTube.

\section{Introdução}

Para os cidadãos interessados em ciência é necessário, fortalecer a cultura e alfabetização científica. Na Ibero América, as pesquisas de percepção publica da ciência e tecnologia (C\&T) são recentes, o primeiro estudo iniciado em 2001 culminou, em 2003, com a aplicação da primeira enquete comparativa sobre a percepção pública da ciência em grandes cidades de quatro países: Campinas (Brasil), Salamanca e Valladolid (Espanha), Buenos Aires (Argentina) e Montevidéu (Uruguai) com o objetivo de desenvolver um padrão metodológico regional e construir indicadores quantitativos que permitissem uma comparação internacional (FAPESP, 2010). Em 
uma nova pesquisa ibero-americana, realizada em 2007, um novo questionário foi aplicado na Colômbia, Argentina, Venezuela, Espanha, Panamá, Chile e Brasil, o questionário abordou quatro temas: 1) informação e interesse sobre ciência e tecnologia; 2) opinião cidadão sobre políticas públicas em ciência e tecnologia; 3) atitudes e avaliações sobre ciência e tecnologia e 4) apropriação social da ciência e tecnologia (OIE, 2009).

Os resultados destes dois estudos mostram-se preocupantes. Sendo observado que os cidadãos não conhecem instituições que se dedicam a fazer pesquisa científica em seu países. Acreditam que os cientistas ganham muito dinheiro mas não consideram prioridade o financiamento em C\&T. À pergunta: "Na sua opinião a profissão científica é atraente para os jovens?" Mais de 50\% de jovens entre 16 e 34 anos de idade respondeu que a profissão científica não é atraente.

Em 2011 foi publicado uma pesquisa ibero-americana realizada na Colômbia, Argentina, Peru, Paraguai, Espanha, Uruguai e Brasil, cujo objetivo geral era proporcionar um panorama da percepção dos estudantes do ensino médio de diversas cidades a temas científicos. Tal pesquisa foi motivada pela preocupação com o declínio da taxa de inscrição em faculdades nas áreas de ciências exatas e naturais, áreas estratégicas para enfrentar os desafios futuros dos países (Elias, C. 2008). À pergunta: “O que você vai estudar?" Apenas o 2,7\% respondeu ciências exatas e naturais. Os principais fatores para não escolher a profissão científica são a dificuldade dos conteúdos da área (60,7\%) e que os conteúdos são muito chatos (50,6\%). (OEI, 2011).

A cultura científica está em crise na Ibero América. A rejeição que as matérias científicas geram, o fracasso escolar nas mesmas por um elevado número de estudantes, o pouco interesse em prosseguir estudos científicos, e resultados ruins em ciência pela maioria dos países iberoamericanos que participaram do PISA (Programme for International Student Assessment), estando todos eles abaixo da média mundial, são sinais desta crise. (OECD, 2012; Asencio, 2012). O baixo nível educacional é relaciona-se com a pobreza, problemas de saúde, elevada mortalidade, procriação descontrolada e muitos outros sinais de fracasso na sociedade (Bazzo, 2012).

E debate atual sobre o declínio da educação, levou o Centro de Altos Estudos Universitários da OEI em 2009 a criação da Comunidade de Educadores Ibero-americanos para a Cultura Científica (CECC), para promover a cultura científica no âmbito educativo e o desejo de introduzir novos materiais e estratégias didáticas (Martín \& Osorio, 2012).

A elaboração e utilização de novos materiais e estratégias didáticas podem ter utilidade para uma aprendizagem de maior qualidade, motivação, interesse e melhor aquisição de conhecimentos. Há uma hipótese (ligada ao modelo de déficit) de que exista uma espécie de círculo virtuoso, em que um maior interesse corresponda à maior busca de informação e 
aquisição de conhecimento, e que isso leve, por sua vez, a atitudes mais positivas e otimistas com respeito à C\&T (Vogt \& Castelfranchi 2009).

O Laboratório de Tecnologia Educacional (LTE) do Instituto de Biologia da Universidade Estadual de Campinas (Brasil) desde 1996 atua no desenvolvimento de produtos educacionais voltados ao ensino e divulgação científicos, com grande acervo de conteúdo disponibilizado para ser usado livremente e, desta forma, levar ferramentas que incentivam a cultura científica dos brasileiros. O material desenvolvido pelo LTE é publicado e distribuído em diversas plataformas de distribuição de conteúdo digital. O presente estudo visa compreender como cada uma das plataformas de distribuição de conteúdo digital contribui para a disseminação do material produzido pelo LTE e levantar discussões sobre quais instrumentos dão maior visibilidade ao material por nós produzido.

\section{Metodologia}

Os dados de uso dos produtos educacionais do Laboratório de Tecnologia Educacional foram tomados a partir de na seguintes plataformas de distribuição de conteúdo digital:

1. Biblioteca Digital de Ciências. http://www.bdc.ib.unicamp.br

2. Portal do professor. http://portaldoprofessor.mec.gov.br/buscaGeral.html?q=galembeck

3. Canal no YouTube. http://www.youtube.com/edgalemb

4. APPs no iTunes Store. https://itunes.apple.com/br/artist/lte-ib-unicamp/id421224798.

5. APPs no Google Play (Antigo Android Market). https://play.google.com/store/apps/developer?id=Eduardo+Galembeck

A Biblioteca Digital de Ciências foi inaugurada de 2006 com a finalidade de concentrar em um único local toda produção do LTE. É uma biblioteca aberta à submissão de softwares, imagens, teses, monografias e apostilas voltadas ao ensino de qualquer disciplina da área biológica. Todo conteúdo disponibilizado é oferecido gratuitamente para ser usado livremente.

O Portal do Professor do Ministério da Educação foi lançado em 2008 em parceria com o Ministério da Ciência e Tecnologia. Tem como objetivo apoiar os processos de formação dos professores e enriquecer a sua prática pedagógica. É um espaço público e pode ser acessado livremente por todos os interessados. A comunidade tem à disposição sugestões de planos de aula, mídias de apoio a aulas, notícias sobre educação e iniciativas do Ministério da Educação (MEC), pode-se ainda compartilhar planos de aula, participar de discussões ou fazer cursos.

Fundado em fevereiro de 2005, o YouTube é um espaço público onde bilhões de pessoas descobrem e compartilham vídeos originais e os assistem. O YouTube oferece um fórum para as pessoas se conectarem, informarem e inspirarem outras pessoas por todo o mundo. Atua como 
uma plataforma de distribuição para criadores de conteúdo original e para grandes e pequenos anunciantes.

iTunes Store foi lançado em 2008 é um serviço para o iPhone, iPod Touch e iPad criado pela Apple Inc. que permite aos usuários navegar e fazer download de aplicativos. Dependendo do aplicativo, ele pode ser gratuito ou pago. Os aplicativos podem ser baixados diretamente no dispositivo (iPhone, iPod e iPad), ou baixados para um computador compatível (Mac).

Google tornou disponível o Android Market em 2008, em 2012 o serviço foi relançado com um novo nome Google Play. Google Play é a loja online para distribuição de aplicativos, jogos, filmes, música e livros. Esta mudança de nome para Google Play ocorreu para que o Google pudesse distribuir todo seu conteúdo digital em um só lugar. Os aplicativos do Google Play estão disponíveis de graça ou a um custo, para o sistema operacional Android. Podem ser baixados diretamente para um dispositivo móvel ou em um computador compatível.

Os dados de utilização dos produtos educacionais foram obtidos a partir das ferramentas próprias disponibilizadas em cada um das plataformas utilizadas. A análise estatística foi feita com o software R. Foram realizadas análises de estatísticas descritivas e testes de normalidade de Shapiro-Wilk que é um teste de ajuste de contraste usado para verificar se os dados são provenientes de uma população com uma distribuição normal. Para o teste do comparação de grupos foi eleito o teste de Kruskal-Wallis que é um método não paramétrico usado para testar se um conjunto de amostras provêm da mesma distribuição, sendo uma extensão do teste $U$ de Mann-Whitney para mais de duas amostras. Para determinar entre quais grupos houve uma diferença estatisticamente significativa foi eleito o teste de comparação múltipla depois de Kruskal-Wallis, este teste ajuda a determinar quais grupos são diferentes com comparações pareadas ajustados de forma adequada. Esses pares de grupos que foram observadas diferenças superior a um valor crítico são considerados estatisticamente diferente a um determinado nível de significância (Matloff, N. 2011).

\section{Resultados e Discussão}

Tipo e número de produtos educacionais disponíveis em cada plataforma de distribuição de conteúdo digital (Tabela. 1). BD refere-se à Biblioteca Digital de Ciência, PP refere-se ao Portal do Professor, YT refere-se ao YouTube, AS refere-se à Apple Store, GP refere-se ao Google Play. Os tipos de produtos A, D, V e S são respectivamente Áudio, Documento, Vídeo e Software. 
Tabela 1 - Tipo e número de produtos educacionais.

\begin{tabular}{ccccccccccc} 
Tipo & BD & $\%$ & PP & $\%$ & YT & $\%$ & AS & $\%$ & GP & $\%$ \\
\hline A & 41 & 28,08 & 45 & 27,27 & 0 & 0 & 0 & 0 & 0 & 0 \\
D & 51 & 34,93 & 43 & 26,06 & 0 & 0 & 0 & 0 & 0 & 0 \\
V & 31 & 21,23 & 31 & 18,79 & 42 & 100 & 0 & 0 & 0 & 0 \\
S & 23 & 15,75 & 46 & 27,88 & 0 & 0 & 11 & 100 & 6 & 100 \\
Total & 146 & 100 & 165 & 100 & 42 & 100 & 11 & 100 & 6 & 100
\end{tabular}

O indicador escolhido para comparações de tempo disponível foi Meses na web (MW). A avaliação do tempo na web foi realizado entre 13 de fevereiro de 2009 e 13 de setembro de 2013. A Tabela 2 mostra o tempo que cada tipo de produto esteve disponível para sua utilização.

Tabela 2 - Meses na web (MW).

\begin{tabular}{ccccccc} 
Tipo & MW & BD & PP & YT & AS & GP \\
\hline \multirow{2}{*}{ A } & Mínimo & 7,30 & 13,58 & & & \\
& Máximo & 54,87 & 36,56 & & & \\
& Média & 36,89 & 27,63 & & & \\
D & Mínimo & 23,61 & 14,76 & & & \\
& Máximo & 23,61 & 36,66 & & & \\
& Média & 23,61 & 22,55 & & & \\
& Mínimo & 18,12 & 16,18 & 4,37 & & \\
& Máximo & 43,30 & 36,33 & 27,75 & 1,64 \\
& Média & 26,85 & 26,03 & 18,57 & & 13,07
\end{tabular}


Ao realizar as comparações da visibilidade dos produtos educacionais desenvolvidos pelo LTE foi eleito como indicador o número de acessos por mês (AM), sendo que essa unidade tem características diferentes em cada um das plataformas estudadas. Nos sistemas baseados na Web, esse indicador é referente ao número de vezes que um material foi acessado, ou seja, que a respectiva URL onde o material está disponível foi acessada por um navegador. Para o material disponível em lojas de distribuição de material online, esse valor se refere ao número de vezes que os aplicativos foram instalados em dispositivos móveis.

O total de produtos educacionais do LTE disponíveis em pelo menos duas das cinco plataformas de distribuição de conteúdo digital foi de 196. O número de acessos total foi 712.446, distribuídas assim 467.218 (65.68\%) Biblioteca Digital de Ciências, 53.135 (7.46\%) Portal do Professor, 91.273 (12.81\%) YouTube, 82.035 (11.51\%) Apple Store 18.785 (2.64\%) Google Play. A Tabela. 3 mostra o número de acessos (NA) e acessos por mês (AM) para cada tipo de produto e plataforma de distribuição de conteúdo digital.

Tabela 3 - Número de acessos (NA) e acessos por mês (AM).

\begin{tabular}{cccccccccccc} 
Tipo & NA & BD & AM & PP & AM & YT & AM & AS & AM & GP & AM \\
\hline \multirow{4}{*}{ A } & mínimo & 890 & 41,16 & 74 & 2,56 & & & & & & \\
& máximo & 6749 & 145,10 & 655 & 26,81 & & & & & & \\
& média & 2691 & 74,42 & 259 & 10,39 & & & & & & \\
D & mínimo & 1219 & 51,64 & 56 & 2,62 & & & & & & \\
& máximo & 14229 & 602,78 & 693 & 20,77 & & & & & & \\
& média & 2605 & 110,35 & 183 & 7,99 & & & & & & \\
\multirow{2}{*}{ V } & mínimo & 1578 & 37,12 & 105 & 5,86 & 182 & 9,90 & & & & \\
& máximo & 4472 & 242,34 & 856 & 52,92 & 18164 & 676,71 & & & & \\
& média & 2524 & 110,81 & 384 & 15,74 & 2173 & 133,60 & & & 150 \\
\multirow{2}{*}{ S } & mínimo & 1225 & 30,54 & 57 & 1,97 & & & 185 & 41,68 & 77 & 15,21 \\
& máximo & 16128 & 360,97 & 1483 & 41,12 & & & 20576 & 878,86 & 13675 & 576,90 \\
& média & 6340 & 146,61 & 472 & 13,65 & & & 7458 & 426,60 & 3131 & 163,20
\end{tabular}

Com o objetivo de definir o teste estatístico para fazer as comparações de visibilidade foi aplicado aos dados o teste de normalidade de Shapiro-Wilk. A exceção do Apple Store, a distribuição foi não-normal. Em seguida, os dados foram submetidos ao teste não paramétrico Kruskal-Wallis para determinar se há diferenças estatisticamente significativas entre as médias dos resultados obtidos. Os resultados obtidos nesse teste têm significância estatística quando o valor de $p$ é inferior a 0.05. Os resultados do teste de Kruskal-Wallis foram significativos ( $p$-value < 2.2e-16), então pelo menos uma das amostras é diferente das outras. 0 teste não identifica onde ocorrem e quantas são as diferenças. Daí a necessidade de utilizar o teste de comparação múltipla depois de Kruskal-Wallis, o qual permitiu identificar as diferenças significativas (Figura 1). 
a.

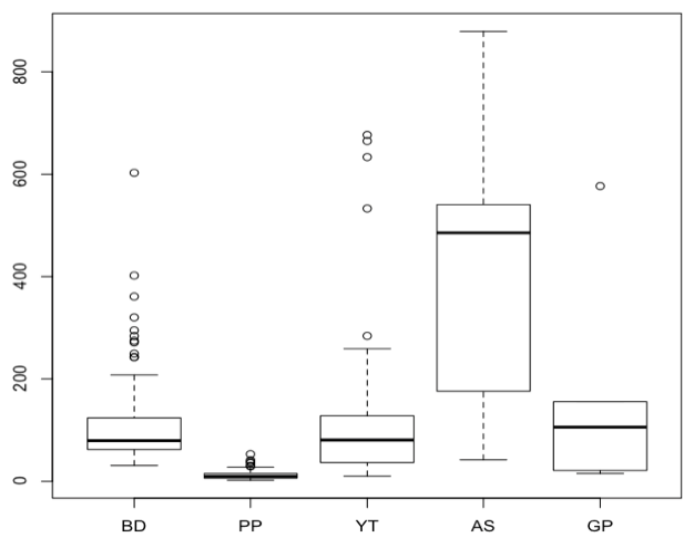

b.

Teste de comparação múltipla depois de Kruskal-Wallis . P. value: 0.05

\begin{tabular}{cccc} 
Comparação & $\begin{array}{c}\text { Diferença } \\
\text { observada }\end{array}$ & $\begin{array}{c}\text { Diferença } \\
\text { critica }\end{array}$ & Diferença \\
\hline AS-BD & 36,05 & 93,88 & Falso \\
AS-GP & 75,27 & 152,34 & Falso \\
AS-PP & 245,03 & 93,50 & Verdadeiro \\
AS-YT & 82,84 & 101,69 & Falso \\
BD-GP & 12,23 & 125,06 & Falso \\
BD-PP & 181,99 & 34,11 & Verdadeiro \\
BD-YT & 19,80 & 52,57 & Falso \\
GP-PP & 169,76 & 124,77 & Verdadeiro \\
GP-YT & 7,58 & 131,03 & Falso \\
PP-YT & 162,19 & 51,89 & Verdadeiro
\end{tabular}

Figura 1 -a) Box plot comparando acessos aos produtos nas cinco plataformas estudadas.

b) Resultados do teste de comparação múltipla depois de Kruskal-Wallis.

Para determinar a visibilidade por tipo de produto educacional foram tomados todos os valores de acessos por mês e depois calculou-se a media para cada tipo de produto (Tabela 4).

Tabela 4 - Relação Produto-Acessos por mês (AM).

Tipo de produto

A

D

V

S
Acessos por mês

40,92

63,53

91,68

112,47

Os resultados do teste de normalidade e comparação de meios foram respectivamente Lilliefors (Kolmogorov-Smirnov) normality test (Audio: 1.322e-09; Documento: 7.723e-15; Vídeo: 2.2e-16e Software: 2.2e-16) e Kruskal-Wallis ( $p$-value $=0.0008012$ ). Resultados do teste de comparação múltipla depois de Kruskal-Wallis se mostram na figura 2. 
a.

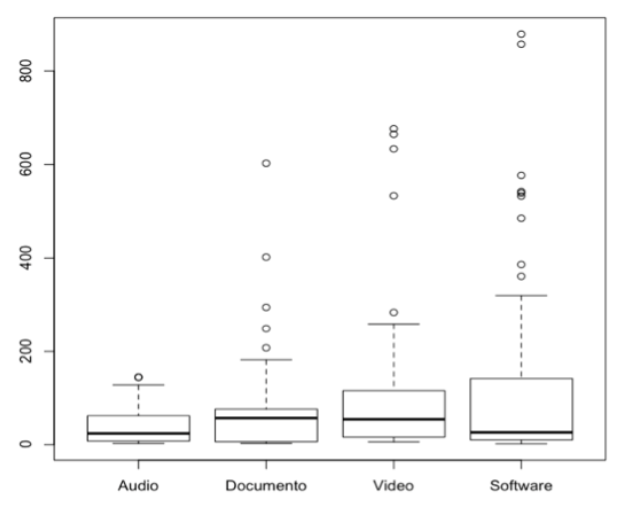

b.

Teste de comparação múltipla depois de Kruskal-Wallis . P. value: 0.05

\begin{tabular}{cccc} 
Comparação & $\begin{array}{c}\text { Diferença } \\
\text { observada }\end{array}$ & $\begin{array}{c}\text { Diferença } \\
\text { critica }\end{array}$ & Diferença \\
\hline A-D & 14,83 & 42,10 & Falso \\
A-S & 33,19 & 43,03 & Falso \\
A-V & 59,84 & 41,13 & Verdadeiro \\
D-S & 18,35 & 42,10 & Falso \\
D-V & 45,01 & 40,16 & Verdadeiro \\
S-V & 26,65 & 41,13 & Falso
\end{tabular}

Figura 2 - a) Box plot comparando visibilidade por tipo de produto educacional.

b) Resultados do teste de comparação múltipla depois de Kruskal-Wallis.

Para determinar a relação custo/visibilidade (C/V), o custo de cada produto educacional foi dividido pelo número de acessos por mês e depois calculou-se a media para cada tipo de produto (Tabela 5). Os resultados do teste de normalidade e comparação de meios foram respectivamente Lilliefors (Kolmogorov-Smirnov) normality test (Audio: 2.693e-09; Documento: 7.723e-15; Vídeo: 2.2e-16 e Software: 1.33e-12) e Kruskal-Wallis ( $p$-vlue $=7.871 \mathrm{e}-07$ ). Resultados do teste de comparação múltipla depois de Kruskal-Wallis se mostram na figura 3.

Tabela 5 - Relação custo- visibilidade (C/V).

\begin{tabular}{cc} 
Tipo de produto & C/V \\
\hline A & 0,41 \\
D & 0,30 \\
V & 0,42 \\
S & 0,37
\end{tabular}


a.

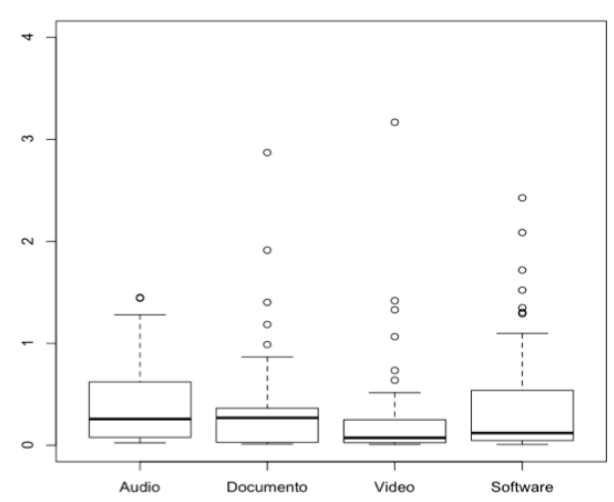

b.

\begin{tabular}{cccc}
\multicolumn{3}{c}{ Teste de comparação múltipla depois de Kruskal-Wallis . P. value: 0.05} \\
Comparação & $\begin{array}{c}\text { Diferença } \\
\text { observada }\end{array}$ & $\begin{array}{c}\text { Diferença } \\
\text { critica }\end{array}$ & Diferença \\
\hline A-D & 52,16 & 42,09 & Verdadeiro \\
A-S & 38,20 & 43,02 & Falso \\
A-V & 85,89 & 41,11 & Verdadeiro \\
D-S & 13,95 & 42,22 & Falso \\
D-V & 33,72 & 40,26 & Falso \\
S-V & 47,68 & 41,24 & Verdadeiro
\end{tabular}

Figura 3 -a) Box plot comparando custo-visibilidade por tipo de produto educacional.

b) Resultados do teste de comparação múltipla depois de Kruskal-Wallis.

O estudo apresentado neste artigo revela que mesmo com variações observadas entre a visibilidade dada ao material publicado na BD, YouTube, Apple App Store a Google Play, não há diferença estatisticamente significativa entre o material publicado nestas plataformas. Nota-se, no entanto, que o Portal do Professor é bastante deficiente com meio de divulgação de objetos educacionais digitais se comparado às outras quatro plataformas.

Sobre a relação custo/visibilidade do tipo de produção do LTE, nota-se que apesar dos vídeos terem uma maior relação custo/visibilidade, essa diferença é estatisticamente significativa apenas em relação aos softwares. O fato do LTE ter um histórico de quase 20 anos de desenvolvimento de software e infraestrutura voltada a esse tipo de mídia, e depender de terceiros em boa parte da produção de vídeos que desenvolvemos, pode ter sido responsável por um maior custo na nossa produção de vídeo se comparada à produção de software, ou seja, o número de acessos aos software ligeiramente superior aos vídeos, aliado ao menor custo na produção dos softwares, faz com que a relação custo/visibilidade para o material desenvolvido pelo LTE seja mais favorável para softwares. Os documentos são os materiais com melhor relação/custo benefício, por terem um custo bastante reduzido em relação aos demais matérias, porém têm menos visibilidade do que vídeos e softwares.

\section{Conclusões}

As análises apresentadas neste artigo nos permitem concluir que a BDC dá visibilidade ao material produzido pelo LTE e nela publicado, similar ao que conseguimos em plataformas como 
YouTube, Google Play e Apple App Store, o que indica que a BDC é um bom meio de divulgação de material educacional digital.

O Portal do Professor é um ambiente que dá menos visibilidade do que plataformas como a BDC, YouTube, Google Play e Apple App Store. Esperamos que essa diferença se reduza agora com a reformulação do Portal, que passou a apresentar uma interface mais amigável e um melhor sistema de busca, indexação e navegação.

Os softwares desenvolvidos pelo LTE são mais acessados se comparados com os programas de áudio, vídeo e documentos. Essa visibilidade alcançada com softwares, aliada à projeção de crescimento de usuários de dispositivos móveis como Tablets e Smartphones apontam para um cenário bastante promissor para o desenvolvimento desta tecnologia com finalidades educacionais e de divulgação científica.

\section{Referências}

Asencio, E. Una alternativa didáctica para el perfeccionamiento del proceso de enseñanzaaprendizaje de las ciencias. Revista Iberoamericana de Educación. N. 58, p. 81-97. 2012.

Bazzo, W. Cultura Científica versus humanística: a cts é o elo? Revista lberoamericana de Educación. N. 5, p. 61-79. 2012.

Elias, Carlos. La razón estrangulada. Madrid: Ramdon Hause Mondadori S.A. 2008.

Vogt, Carlos. Percepção pública da ciência e tecnologia no estado de são Paulo . In Indicadores de Ciência, Tecnologia e Inovação em São Paulo 2010. São Paulo: Centro da documentação e Informação da FAPESP. 2010. Cap. 12, p. 1-51.

Martín Gordillo, M \& Osorio, C. Comunidad de educadores iberoamericanos para la Cultura Científica. una red para la innovación. Revista Iberoamericana de Educación. N, 58. p. 193-218. 2012.

Matloff, Norman. The art of $\mathbf{R}$ programming: tour of statistical software design. San Francisco. No starch press, Inc. 2011.

Organisation for Economic Co-operation and Development (OECD). 2012. PISA 2012 Results in Focus What 15-year-olds know and what they can do with what they know. Disponivel em:< http://www.oecd.org/pisa/keyfindings/pisa-2012-results-overview.pdf>. Acesso em: 03 Jun. 2014. Organización de Estados Iberoamericanos (OEI). 2009. Cultura científica en Iberoamérica. Encuesta en grandes núcleos urbanos. Disponível em:< http://www.oei.es/salactsi/CulturaCientificaEnlberoamerica.pdf >. Acesso em: 03 Jun. 2014. 
Organización de Estados Iberoamericanos (OEI). Carmelo Polino (Compilador). 2011. Los estudiantes y la ciencia : encuesta a jóvenes iberoamericanos. Disponível em:< http://www.oei.es/salactsi/libro-estudiantes.pdf >. Acesso em: 03 Jun. 2014.

Vogt, C \& Castelfranchi, J. Interesse, informação e comunicação. Cultura científica. In Iberoamérica. Encuesta en grandes núcleos urbanos. Madrid. Gráficas Lizarra, S.L. 2009. p. 21-36.

Eduardo Galembeck - Doutor em Ciências pela Universidade Estadual de Campinas (UNICAMP). Professor do Programa de Pós-Graduação em Biologia Funcional e Molecular e do Programa de Pós-Graduação Multiunidades em Ensino de Ciências e Matemática (UNICAMP). Bolsista de Produtividade em Desenvolvimento Tecnológico e Extensão Inovadora do CNPq. E-mail: eg@unicamp.br

Juan Carlos Vega Garzon - Mestre em Microbiologia (Universidad Nacional de Colombia). Doutorando do Programa de Pós-Graduação em Biologia Funcional e Molecular (UNICAMP). Email: jcvegag@unal.edu.co 